\title{
Patient Safety in Primary Care
}

V Pedrosa $\mathrm{M}$

European Journal of Public Health, Volume 30, Issue

Supplement_2, June 2020, ckaa040.061,

https://doi.org/10.1093/eurpub/ckaa040.061

Published: 24 June 2020

\begin{abstract}
Introduction Patient safety and the development of quality safety processes have been gaining prominence in business management in the public and political sector, particularly in Europe, after the Quality and Safety in European Hospitals project was driven by the support of the Lisbon strategy agenda. Scientific research has also followed this trend. However, the study of patient safety management and methods is still scarce.

Objectives Resorting to Cartaxo Health Centre, the research contributes to the analysis of the proposition that there is not only a lack of knowledge of the professionals who directly contact the patient about the patients safety in primary health care, but also that the methods used in practice are very intuitive and individualised by the absence of formal and institutional coordinated orientation.
\end{abstract}

Methodology Using a qualitative methodology and the case study method, a semi-structured interview was conducted with 40 health professionals - among doctors, nurses, and technicians - who contacted patients, in order to identify what they know and consequently incorporate about patient safety. The interviews were conducted between February and June 2015

Results We confirmed several discrepancies between functional units. It was clarified that in the domicile the knowledge is scarcer.

Conclusion We have delimited factors that do not ensure a reliable environment and culture, to improve with a training plan and institutional coordination that supports notification. The complexity of the context impacts on the 
nature and dimension of adverse events due to stress, workload and organization. A Taxonomy of Adverse Events was constructed and its path from the source to the solution was mapped. It's our intention to share this Taxonomy with the professionals, with a strong expectation that will improve Quality and Safety in Portuguese Primary Care.

Topic: health personnel, nurses, primary health care, safety, workload, taxonomic classification, stress, coordination, patient safety, quality improvement, adverse event, agenda, portuguese

Issue Section: Oral Communications

This content is only available as a PDF.

(c) The Author(s) 2020. Published by Oxford University Press on behalf of the European Public Health Association. All rights reserved.

This article is published and distributed under the terms of the Oxford University Press, Standard Journals Publication Model (https://academic.oup.com/journals/pages/open_access/funder_policies/c horus/standard_publication_model)

You do not currently have access to this article.

\section{Comments}

0 Comments

\section{Sign in}

Don't already have an Oxford Academic account? Register 